\title{
SADDLEPOINT APPROXIMATIONS TO SENSITIVITIES \\ OF TAIL PROBABILITIES OF RANDOM SUMS AND COMPARISONS WITH MONTE CARLO ESTIMATORS
}

\author{
Riccardo Gatto ${ }^{\dagger}$ and Chantal Peeters ${ }^{\ddagger}$
}

Submitted: January 2013

Revised: June 2013

\begin{abstract}
This article proposes computing sensitivities of upper tail probabilities of random sums by the saddlepoint approximation. The considered sensitivity is the derivative of the upper tail probability with respect to the parameter of the summation index distribution. Random sums with Poisson or Geometric distributed summation indices and Gamma or Weibull distributed summands are considered. The score method with importance sampling is considered as an alternative approximation. Numerical studies show that the saddlepoint approximation and the method of score with importance sampling are very accurate. But the saddlepoint approximation is substantially faster than the score method with importance sampling. Thus the suggested saddlepoint approximation can be conveniently used in various scientific problems.
\end{abstract}

\section{Key words and phrases}

Change of measure, exponential tilt, Gamma distribution, Geometric distribution, importance sampling, Poisson distribution, rare event, score Monte Carlo method, Weibull distribution.

2010 Mathematics Subject Classification: 41A60, $65 \mathrm{C} 05$.

$\dagger$ Institute of Mathematical Statistics and Actuarial Science, University of Bern, Alpeneggstrasse 22, 3012

Bern, Switzerland. Email: gatto@stat.unibe.ch.

†Email: chantal peeters@hotmail.ch.

The authors are grateful to the Editor, the Associate Editor and two anonymous Reviewers, for thoughtful comments, suggestions and corrections which improved the quality of the article. Riccardo Gatto is grateful to the Swiss National Science Foundation for financial support (grant 200021-121901). 


\section{Introduction}

Let $(\Omega, \mathcal{F}, m)$ be a measure space, where $\mathcal{F} \subset \mathcal{P}(\Omega)$ is a $\sigma$-algebra, and let $\left\{\mathrm{P}_{\theta}\right\}_{\theta \in \Theta}$ be a class of probabilities measures such that $\mathrm{P}_{\theta} \ll m, \forall \theta \in \Theta$, where $\Theta$ is an open interval of $\mathbb{R}$. Let $X_{1}, X_{2}, \ldots$ be independent random variables on $(\Omega, \mathcal{F})$ with distribution function (d.f.) $F_{X}$ independent of $\theta$, survivor function $\bar{F}_{X}=1-F_{X}$ and density $f_{X}$, with respect to (w.r.t.) the Lebesgue measure on $\mathbb{R}$. Let $N$ be an independent $\mathbb{N}$-valued random variable on $(\Omega, \mathcal{F})$ with density $p_{\theta}$, w.r.t. the counting measure, depending on $\theta \in \Theta$. Here $\mathbb{N} \stackrel{\text { def }}{=}\{0,1, \ldots\}$. We consider the random sum $S=\sum_{k=0}^{N} X_{k}$, where $X_{0} \stackrel{\text { def }}{=} 0$, and the upper tail probability

$$
z(\theta ; x) \stackrel{\text { def }}{=} \mathrm{P}_{\theta}[S \geq x]=p_{\theta}(0) \mathrm{I}\{x \leq 0\}+\sum_{k=1}^{\infty} \int_{\mathbb{R}^{k}} \mathrm{I}\left\{\sum_{j=1}^{k} x_{j} \geq x\right\} \prod_{j=1}^{k} f_{X}\left(x_{j}\right) \mathrm{d} x_{j} p_{\theta}(k),
$$

$\forall \theta \in \Theta$, where $x \in \mathbb{R}$ is large and where $\mathrm{I}\{A\}$, or alternatively $\mathrm{I}_{A}$, denote the indicator of statement $A$. In this case, $z(\theta ; x)$ is typically small and referred as a rare event probability. The goal is to approximate accurately the sensitivity w.r.t. $\theta$ of this rare event probability, namely to approximate

$$
z^{\prime}(\theta ; x) \stackrel{\text { def }}{=} \frac{\partial}{\partial \theta} z(\theta ; x)=\frac{\partial}{\partial \theta} \mathrm{P}_{\theta}[S \geq x], \forall \theta \in \Theta
$$

where $x \in \mathbb{R}$ is large.

Random sums with Poisson or Geometric distributed counts $(N)$ and positive summands $\left(X_{1}, X_{2}, \ldots\right)$ are often used to model phenomena occurring e.g. in earth sciences, ecology, biology, insurance and finance. In insurance, the insurer total claim amount generated by a portfolio of risks over a fixed period is often represented by a Poisson random sum. Further, let $Y_{1}, Y_{2}, \ldots$ be $\mathbb{R}_{+}^{*}$-valued independent individual claim amounts with d.f. $F_{Y}$ and expectation $\mu_{Y} \in \mathbb{R}_{+}^{*}$. Here $\mathbb{R}_{+}^{*} \stackrel{\text { def }}{=}(0, \infty)$. Let $K_{t}$ be a Poisson process with rate $\lambda>0$ at a given time $t \geq 0$ and let $Z_{t}=\sum_{k=0}^{K_{t}} Y_{k}$, where $Y_{0} \stackrel{\text { def }}{=} 0$, be the total claim amount at time $t \geq 0$. The compound Poisson risk process of the insurer financial reserve at time $t \geq 0$ is given by $U_{t}=x+c t-Z_{t}$, where $x \geq 0$ is the initial capital and $c>0$ is the premium rate. The probability of ruin is the probability that $\left\{U_{t}\right\}_{t \geq 0}$ ever falls below the null line. A classical result is the re-expression of the probability of ruin as $z(\theta ; x)$ given in (1), where $N$ has the Geometric distribution with parameter $\theta=\lambda \mu_{Y} / c<1$ and the summands have d.f. $F_{X}(x)=\int_{0}^{x}\left[1-F_{Y}(y)\right] d y / \mu_{Y}, \forall x \geq 0$. Thus the sensitivity of the probability of ruin w.r.t. $\theta$ is given by $z^{\prime}(\theta ; x)$ in $(2)$. Some standard references on risk processes are, for example, Asmussen (2000), Bühlmann (1970), Gerber (1979) and Mikosch (2004).

Finding analytical and accurate approximations to the distribution of a random sum, or to the distribution of a compound process at a given time, has been investigated by several authors. We mention some of them. Esscher $(1932,1963)$ proposed an approximation based on the exponential tilt (or the Esscher transform), which is the essence of both saddlepoint approximation and importance sampling. Willmot (1989) provided an infinite series representation of the density of the insurer total claim amount. Jensen (1991) proposed saddlepoint approximations for homogeneous compound Poisson processes with 
Wiener perturbation, under force of interest and under Markov modulation (i.e. switching between different compound Poisson processes). Gatto (2011) provided saddlepoint approximations to tail probabilities, values at risk and tail values at risk of the doubly compound insurer total claim amount, which is based on a primary counting birth process, for the number of catastrophic events, and on a secondary counting distribution, for the number of individual losses generated from each catastrophe of the primary process. Gatto (2012) provided saddlepoint approximations to tail probabilities and quantiles of the compound Poisson process under force of interest and under time inhomogeneity, with several types of periodic intensity functions.

However, the problem of computing analytically the random sum sensitivity $z^{\prime}(\theta ; x)$ has not received much attention. Available methods rely on Monte Carlo simulation, see Asmussen and Glynn (2007), Chapter VII. For situations where $x$ is large, the method of score with importance sampling can used to approximate $z^{\prime}(\theta ; x)$ accurately, see Asmussen and Rubinstein (1999), Asmussen and Glynn (2007), p. 239-240, and Section 3 of the present article.

A simple analytic approximation to $z^{\prime}(\theta ; x)$ could be derived from the limiting normal approximation. Denote by $\left\{S_{\theta}\right\}_{\theta \in \Theta}$ a stochastic process on $(\Omega, \mathcal{F}, \mathrm{P})$ such that the distribution of $S_{\theta}$ under $\mathrm{P}$ is the distribution of $S$ under $\mathrm{P}_{\theta}, \forall \theta \in \Theta$. Let $\bar{\theta}=\sup \Theta$ or $\inf \Theta$, according to the selected model, let $\mathrm{E}$ and var be the expectation and variance under $\mathrm{P}$ and assume

$$
\frac{S_{\theta}-\mathrm{E}\left[S_{\theta}\right]}{\sqrt{\operatorname{var}\left(S_{\theta}\right)}} \stackrel{\mathrm{d}}{\longrightarrow} \mathcal{N}(0,1), \text { as } \theta \rightarrow \bar{\theta}
$$

Then

$$
z(\theta ; x) \simeq z_{\mathrm{a}}(\theta ; x) \stackrel{\text { def }}{=} \simeq 1-\Phi\left(\frac{x-\mathrm{E}\left[S_{\theta}\right]}{\sqrt{\operatorname{var}\left(S_{\theta}\right)}}\right),
$$

where $\simeq$ means approximately equal. A rough approximation to the sensitivity $z^{\prime}(\theta ; x)$ could be obtained by differentiating the last expression. However, it is well-known that asymptotic normal approximations often lead to inaccurate approximations to small tail probabilities, as they often entail large relative errors. Clearly, $z(\theta ; x) \rightarrow 0$, as $x \rightarrow \infty$, $\forall \theta \in \Theta$. As explained at the beginning of Section 3,

$$
\liminf _{x \rightarrow \infty} \frac{\log \left|z^{\prime}(\theta ; x)\right|}{\log z(\theta ; x)} \geq 1, \quad \forall \theta \in \Theta,
$$

which means that $z(\theta ; x)$ and $z^{\prime}(\theta ; x)$ have similar logarithmic asymptotics, as $x \rightarrow \infty$. So both $z(\theta ; x)$ and $z^{\prime}(\theta ; x)$ are asymptotically vanishing quantities and so for none of them one should expect accurate normal approximations.

Alternative approximations to sensitivities with small relative errors are thus required and the aims of this article are: to propose an analytical approximation obtained from the saddlepoint approximation, then to illustrate its high numerical accuracy and finally to compare it with an accurate Monte Carlo competitor, namely with the method of score with importance sampling.

The remaining part of this article is organized as follows. In Section 2 we give an approximation to the sensitivity of a random sum obtained from the saddlepoint approxi- 
mation of Lugannani and Rice (1980). Specific results for the Poisson random sum are given under Section 2.1 and for the Geometric random sum under Section 2.2. In Section 3 we summarize the score with importance sampling Monte Carlo method and then we present numerical comparisons: Poisson random sums with Gamma and Weibull summands, in Section 3.1, and Geometric random sums with Gamma summands, in Section 3.2. These numerical comparisons show that the saddlepoint approximation to sensitivities is very accurate and provide thus an efficient alternative to importance sampling simulation. A conclusion is given in Section 4 .

\section{Saddlepoint approximations to sensitivities}

The saddlepoint approximation or method of steepest descent is an important technique of asymptotic analysis, which provides approximations to some types of complex integrals, see e.g. de Bruijn (1981), Chapter 5. It was introduced in statistics by Daniels (1954), who provided the saddlepoint expansion of the density of the sample mean of $n$ independent and identically distributed random variables. The relative error of the first term of the expansion is $\mathrm{O}\left(n^{-1}\right)$, as $n \rightarrow \infty$. Lugannani and Rice (1980) provided a saddlepoint approximation to the survivor function, also with relative error $\mathrm{O}\left(n^{-1}\right)$, as $n \rightarrow \infty$, see also Daniels (1987). Let us define the cumulant generating function (c.g.f.) of $S$ as $K_{\theta}(v)=\log \mathrm{E}_{\mathrm{P}_{\theta}}\left[e^{v S}\right]$, which is assumed existing for all $v$ over a neighborhood of zero. Let us denote by $\phi$ the standard normal density. Lugannani and Rice's (1980) saddlepoint approximation to $z(\theta ; x)=\mathrm{P}_{\theta}[S \geq x]$ takes the form of

$$
z_{\mathrm{s}}(\theta ; x)=1-\Phi\left(r_{x}\right)-\phi\left(r_{x}\right)\left(\frac{1}{r_{x}}-\frac{1}{s_{x}}\right)
$$

where

$$
r_{x}=\operatorname{sgn}\left(v_{x}\right)\left\{2\left[v_{x} x-K_{\theta}\left(v_{x}\right)\right]\right\}^{\frac{1}{2}}, \quad s_{x}=v_{x}\left\{K_{\theta}^{\prime \prime}\left(v_{x}\right)\right\}^{\frac{1}{2}}
$$

and $v_{x}$ is the saddlepoint, i.e. the solution in $v$ of the equation

$$
K_{\theta}^{\prime}(v)=x
$$

for $x$ in the range of $S$. Both $1 / r_{x}$ and $1 / s_{x}$ become arbitrarily large as $x$ approaches $\mathrm{E}_{\mathrm{P}_{\theta}}[S]$, so Lugannani and Rice's approximation can be numerically misleading for $x$ over small neighborhoods of $\mathrm{E}_{\mathrm{P}_{\theta}}[S]$.

We suggest approximating $z^{\prime}(\theta ; x)$ by differentiating the saddlepoint approximation (5), precisely by

$$
\begin{aligned}
z_{\mathrm{s}}^{\prime}(\theta ; x) & \stackrel{\text { def }}{=} \frac{\partial}{\partial \theta} z_{\mathrm{s}}(\theta ; x) \\
& =-\phi\left(r_{x}\right)\left[\frac{r_{x}}{s_{x}} \frac{\partial}{\partial \theta} r_{x}-\frac{1}{r_{x}^{2}} \frac{\partial}{\partial \theta} r_{x}+\frac{1}{s_{x}^{2}} \frac{\partial}{\partial \theta} s_{x}\right],
\end{aligned}
$$


where

$$
\begin{aligned}
\frac{\partial}{\partial \theta} s_{x} & =\frac{\partial}{\partial \theta} v_{x} \cdot \sqrt{K_{\theta}^{\prime \prime}\left(v_{x}\right)}+v_{x} \cdot \frac{\frac{\partial}{\partial \theta}\left\{K_{\theta}^{\prime \prime}\left(v_{x}\right)\right\}}{2 \sqrt{K_{\theta}^{\prime \prime}\left(v_{x}\right)}} \quad \text { and } \\
\frac{\partial}{\partial \theta} r_{x} & =\frac{1}{r_{x}}\left(\frac{\partial}{\partial \theta} v_{x} \cdot K_{\theta}^{\prime}\left(v_{x}\right)-\frac{\partial}{\partial \theta}\left\{K_{\theta}\left(v_{x}\right)\right\}\right) .
\end{aligned}
$$

These derivatives are obtained by using (6) and by assuming $\partial /(\partial \theta) \operatorname{sgn} v_{x}=0$. This last assumption holds whenever $x$ is not close to $\mathrm{E}_{\mathrm{P}_{\theta}}[S]$ and this is true with small tail probabilities.

We should note that Lugannani and Rice (1980) approximation (5) holds when the true distribution is alsolutely continuous and this is not the case here, because $S$ has a probability mass at zero. Formally, one could first apply the saddlepoint approximation to the conditional distribution of $S$ given $N>0$ and then adjust the approximation obtained, as done e.g. in Gatto (2011). This modification would lead to a substantially more complicated derivative w.r.t. $\theta$ and therefore we apply here the saddlepoint approximation directly to the distribution of $S$. This turns out to be very accurate for upper tail probabilities. For the Poisson random sum with Gamma summands presented in Section 2.1 and Example 3.1.1, with parameters $\theta=1, \alpha=2$ and $\beta=1$, we find that for $x>\mathrm{E}_{\mathrm{P}_{\theta}}[S]$, the relative errors $\left\{z_{\mathrm{s}}(\theta ; x)-z(\theta ; x)\right\} / z(\theta ; x)$ decrease with $x$. For example, the approximation to $z(1 ; 3)=0.2673$ has relative error 0.0091 and the approximation $z(1 ; 19)=0.0001$ has relative error 0.0058 . One could explain this high accuracy as follows. Let $f_{S}$ denote the defective density of $S$ over $\mathbb{R}_{+}^{*}$, corresponding to the absolutely continuous part of $S$. Thus the m.g.f. of $S$ is $M_{\theta}(v)=p_{\theta}(0)+\int_{0}^{\infty} e^{v x} f_{S}(x) \mathrm{d} x$. Consider $h>0$ small, then by spreading $p_{\theta}(0)$ uniformly over $[0, h), S$ inherits the (proper) density $f_{S, h}(x) \stackrel{\text { def }}{=} p_{\theta}(0) h^{-1} \mathrm{I}\{x \in[0, h)\}+f_{S}(x), \quad \forall x \geq 0$. The corresponding m.g.f. is

$$
M_{\theta, h}(v) \stackrel{\text { def }}{=} \int_{0}^{\infty} e^{v x} f_{S, h}(x) \mathrm{d} x=p_{\theta}(0)\{1+\mathrm{o}(1)\}+\int_{0}^{\infty} e^{v x} f_{S}(x) \mathrm{d} x=M_{\theta}(v)+\mathrm{o}(1),
$$

as $h \rightarrow 0$. Thus, $M_{\theta}$ is arbitrarily close to the m.g.f. $M_{\theta, h}$ of an absolutely continuous random variable (whose distribution differs only over arbitrarily small intervals, starting from zero). Practically, inverting $M_{\theta}$ instead of $M_{\theta, h}$ is unessential if upper tail probabilities are desired.

\subsection{Approximations for Poisson random sums}

We now consider the Poisson random sum where $N$ is Poisson distributed with parameter $\theta \in \Theta \stackrel{\text { def }}{=} \mathbb{R}_{+}^{*}$, precisely $p_{\theta}(n)=\mathrm{P}_{\theta}[N=n]=e^{-\theta} \theta^{n} / n !, \forall n \in \mathbb{N}$. We assume that $M_{X}(v)=\mathrm{E}_{\mathrm{P}_{\theta}}\left[e^{v X_{1}}\right]$ exists for all $v$ over a neighborhood of zero. The c.g.f. of $S$ is given by

$$
K_{\theta}(v)=\theta\left[M_{X}(v)-1\right]
$$

which exists for all $v$ over a neighborhood of zero. In what follows we assume $v$ in the region of existence of $K_{\theta}(v)$. Differentiating (8) w.r.t. $v$ yields $K_{\theta}^{\prime}(v)=\theta M_{X}^{\prime}(v)$ and $K_{\theta}^{\prime \prime}(v)=\theta M_{X}^{\prime \prime}(v)$. So the saddlepoint equation (6) becomes

$$
\theta M_{X}^{\prime}(v)-x=0 .
$$


Embrechts et al. (1985) showed that for a compound Poisson sum, the relative error of the saddlepoint approximation vanishes as $x \rightarrow \infty$. Intuitively, the saddlepoint approximation at $x$ can be seen as a normal approximation under the exponentially tilted measure with tilting parameter $v_{x}$. Under this new measure, $N$ is Poisson distributed with parameter $\theta_{v_{x}}=\theta M_{X}\left(v_{x}\right)$, so its expectation tends to infinity as $x \rightarrow \infty$; see (21) and (22) for more details. Therefore, a vanishing relative error can be expected.

Differentiating (9) w.r.t. $\theta$ yields

$$
\frac{\partial}{\partial \theta} v_{x}=-\frac{M_{X}^{\prime}\left(v_{x}\right)}{\theta M_{X}^{\prime \prime}\left(v_{x}\right)}=-\frac{x}{\theta^{2} M_{X}^{\prime \prime}\left(v_{x}\right)} .
$$

From (8), (9) and (10) we can obtain the derivatives

$$
\begin{aligned}
& \frac{\partial}{\partial \theta}\left\{K_{\theta}\left(v_{x}\right)\right\}=M_{X}\left(v_{x}\right)-1+\theta \cdot M_{X}^{\prime}\left(v_{x}\right) \frac{\partial}{\partial \theta} v_{x}=M_{X}\left(v_{x}\right)-1-\frac{x^{2}}{\theta^{2} M_{X}^{\prime \prime}\left(v_{x}\right)} \quad \text { and } \\
& \frac{\partial}{\partial \theta}\left\{K_{\theta}^{\prime \prime}\left(v_{x}\right)\right\}=M_{X}^{\prime \prime}\left(v_{x}\right)+\theta \cdot M_{X}^{\prime \prime \prime}\left(v_{x}\right) \frac{\partial}{\partial \theta} v_{x}=M_{X}^{\prime \prime}\left(v_{x}\right)-\frac{x M_{X}^{\prime \prime \prime}\left(v_{x}\right)}{\theta M_{X}^{\prime \prime}\left(v_{x}\right)}
\end{aligned}
$$

which allow to compute $z_{\mathrm{s}}^{\prime}(\theta ; x)$ given by $(7)$.

\subsection{Approximations for Geometric random sums}

We now consider the geometric random sum where $N$ has the geometric distribution with parameter $\theta \in \Theta \stackrel{\text { def }}{=}(0,1)$, precisely $p_{\theta}(n)=\mathrm{P}_{\theta}[N=n]=\theta(1-\theta)^{n}, \forall n \in \mathbb{N}$. We assume that $M_{X}(v)=\mathrm{E}_{\mathrm{P}_{\theta}}\left[e^{v X_{1}}\right]$ exists for all $v$ over a neighborhood of zero. Thus the c.g.f. of $S$ is given by

$$
K_{\theta}(v)=\log \mathrm{E}_{\mathrm{P}_{\theta}}\left[e^{v S}\right]=\log \frac{\theta}{1-(1-\theta) M_{X}(v)},
$$

$\forall v \in \mathbb{R}$ such that $M_{X}(v)<1 /(1-\theta)$. This condition describes an interval including a neighborhood of zero whenever $M_{X}(v)$ exists for all $v$ over a neighborhood of zero. In what follows we assume $v$ in the region of existence of $K_{\theta}(v)$. Differentiating (11) w.r.t. $v$ yields

$$
\begin{aligned}
K_{\theta}^{\prime}(v) & =\frac{(1-\theta) M_{X}^{\prime}(v)}{1-(1-\theta) M_{X}(v)} \quad \text { and } \\
K_{\theta}^{\prime \prime}(v) & =\frac{(1-\theta) M^{\prime \prime}(\nu)}{1-(1-\theta) M_{X}(v)}+\frac{\left(1-\theta^{2}\left[M_{X}^{\prime}(v)\right]^{2}\right.}{\left[1-(1-\theta) M_{X}(v)\right]^{2}} .
\end{aligned}
$$

From (12), the saddlepoint equation (6) can be simplified to

$$
(1-\theta) M_{X}^{\prime}(v)+x(1-\theta) M_{X}(v)-x=0 .
$$

Embrechts et al. (1985) showed that for a Geometric sum, the relative error of the saddlepoint approximation vanishes as $x \rightarrow \infty$. Intuitively, under the tilted measure, $N$ has the Geometric distribution with parameter $\theta_{v_{x}}=1-(1-\theta) M_{X}\left(v_{x}\right)$ and so it has expectation $\left(1-\theta_{v_{x}}\right) / \theta_{v_{x}}$, which tends to infinity as $x \rightarrow \infty$; see (31) and (32) for more details. Therefore a vanishing relative error is expected. 
Differentiating (14) w.r.t. $\theta$ yields

$$
-M_{X}^{\prime}\left(v_{x}\right)+(1-\theta) M_{X}^{\prime \prime}\left(v_{x}\right) \frac{\partial}{\partial \theta} v_{x}-x M_{X}\left(v_{x}\right)+x(1-\theta) M_{X}^{\prime}\left(v_{x}\right) \frac{\partial}{\partial \theta} v_{x}=0
$$

and thus

$$
\frac{\partial}{\partial \theta} v_{x}=\frac{x M_{X}\left(v_{x}\right)+M_{X}^{\prime}\left(v_{x}\right)}{(1-\theta)\left[M_{X}^{\prime \prime}\left(v_{x}\right)+x M_{X}^{\prime}\left(v_{x}\right)\right]} .
$$

From (11) and (13) we obtain

$$
\begin{aligned}
\frac{\partial}{\partial \theta}\left\{K_{\theta}\left(v_{x}\right)\right\}= & \frac{1}{\theta}+\frac{(1-\theta) M_{X}^{\prime}\left(v_{x}\right) \frac{\partial}{\partial \theta} v_{x}-M_{X}\left(v_{x}\right)}{1-(1-\theta) M_{X}\left(v_{x}\right)} \text { and } \\
\frac{\partial}{\partial \theta}\left\{K_{\theta}^{\prime \prime}\left(v_{x}\right)\right\}= & \frac{(1-\theta) M_{X}^{\prime \prime \prime}\left(v_{x}\right) \frac{\partial}{\partial \theta} v_{x}-M_{X}^{\prime \prime}\left(v_{x}\right)}{1-(1-\theta) M_{X}\left(v_{x}\right)} \\
& +\frac{(1-\theta) M_{X}^{\prime \prime}\left(v_{x}\right)\left[(1-\theta) M_{X}^{\prime}\left(v_{x}\right) \frac{\partial}{\partial \theta} v_{x}-M_{X}\left(v_{x}\right)\right]}{\left[1-(1-\theta) M_{X}\left(v_{x}\right)\right]^{2}} \\
& +\frac{2(1-\theta) M_{X}^{\prime}\left(v_{x}\right)\left[(1-\theta) M_{X}^{\prime \prime}\left(v_{x}\right) \frac{\partial}{\partial \theta} v_{x}-M_{X}^{\prime}\left(v_{x}\right)\right]}{\left[1-(1-\theta) M_{X}\left(v_{x}\right)\right]^{2}} \\
& +\frac{2(1-\theta)^{2}\left[M_{X}^{\prime}\left(v_{x}\right)\right]^{2}\left[(1-\theta) M_{X}^{\prime}\left(v_{x}\right) \frac{\partial}{\partial \theta} v_{x}-M_{X}\left(v_{x}\right)\right]}{\left[1-(1-\theta) M_{X}\left(v_{x}\right)\right]^{3}}
\end{aligned}
$$

which allow to compute $z_{\mathrm{s}}^{\prime}(\theta ; x)$ given by $(7)$.

\section{Comparisons with importance sampling estimators of sensitivities}

Generally, let $A(x)$ be a rare event and $Z(x)=\mathrm{I}_{A(x)}$ an estimator of $z(\theta ; x)=\mathrm{P}_{\theta}[A(x)]=$ $\mathrm{E}_{\mathbf{P}_{\theta}}[Z(x)], \forall x>0, \theta \in \Theta$. By rare event is meant that $z(\theta ; x) \rightarrow 0$, as $x \rightarrow \infty, \forall \theta \in \Theta$. We consider the sensitivity $z^{\prime}(\theta ; x)=\partial /(\partial \theta) z(\theta ; x)=\partial /(\partial \theta) \mathrm{E}_{\mathrm{P}_{\theta}}[Z(x)]$. Let $\tilde{\mathrm{P}}$ be another probability measure over $(\Omega, \mathcal{F})$ such that, $\forall \theta \in \Theta, \mathrm{P}_{\theta} \ll \tilde{\mathrm{P}}$. The Radon-Nikodym derivative $\mathrm{dP}_{\theta} / \mathrm{d} \tilde{\mathrm{P}}$ provides the correction factor for the change of measure of integration,

$$
z(\theta ; x)=\mathrm{E}_{\mathrm{P}_{\theta}}[Z(x)]=\mathrm{E}_{\tilde{\mathrm{P}}}\left[Z(x) \frac{\mathrm{dP}_{\theta}}{\mathrm{d} \tilde{\mathrm{P}}}\right] .
$$

Assuming change of differentiation and integration orders without effect, we have

$$
z^{\prime}(\theta ; x)=\mathrm{E}_{\tilde{\mathrm{P}}}\left[Z(x) \frac{\partial}{\partial \theta} \frac{\mathrm{dP}_{\theta}}{\mathrm{dP}}\right]
$$

General conditions for the change of differentiation and integration orders can be found e.g. at p. 222-223 of Asmussen and Glynn (2007). In order to compute $z^{\prime}\left(\theta_{0} ; x\right)$, for some $\theta_{0} \in \Theta$, we can choose $\tilde{P}=P_{\theta_{0}}$. Let $f_{\theta}$ be the density of $\mathrm{P}_{\theta}$ w.r.t. $m$, i.e. $f_{\theta}=\mathrm{dP}_{\theta} / \mathrm{d} m$, $\forall \theta \in \Theta$, then we define the score function at $\theta_{0}$ as

$$
\Psi\left(\theta_{0}\right)=\left.\frac{\partial}{\partial \theta} \frac{\mathrm{dP}_{\theta}}{\mathrm{dP}_{\theta_{0}}}\right|_{\theta=\theta_{0}}=\left.\frac{\partial}{\partial \theta} \frac{f_{\theta}}{f_{\theta_{0}}}\right|_{\theta=\theta_{0}}=\left.\frac{\partial}{\partial \theta} \log f_{\theta}\right|_{\theta=\theta_{0}} .
$$


Thus we obtain the score estimator of the sensitivity with

$$
z^{\prime}\left(\theta_{0} ; x\right)=\mathrm{E}_{\mathrm{P}_{0}}\left[Z(x) \Psi\left(\theta_{0}\right)\right]
$$

see e.g. Rubinstein (1986). The Monte Carlo method of score consists in generating a large number of times the random variable inside the expectation of (15) and in taking the mean of all generated values. In the case of $A(x)=\{S \geq x\}$, where $S$ is the random sum with random index $N$ whose distribution depends on $\theta$, the score is given by

$$
\Psi\left(\theta_{0}\right)=\frac{\left.\frac{\partial}{\partial \theta} p_{\theta}(N)\right|_{\theta=\theta_{0}}}{p_{\theta_{0}}(N)}=\left.\frac{\partial}{\partial \theta} \log p_{\theta}(N)\right|_{\theta=\theta_{0}} .
$$

Provided $\mathrm{E}_{\mathrm{P}_{\theta}}\left[|\Psi(\theta)|^{q}\right]<\infty, \forall q<\infty$, the logarithmic asymptotics statement (4) holds. The justification, which can be found e.g. at p. 239 of Asmussen (2007), follows directly from Hölder's inequality. Indeed, $\forall p, q>1$ such that $1 / p+1 / q=1$, we have

$$
\left|z^{\prime}(\theta ; x)\right|=\left|\mathrm{E}_{\mathrm{P}_{\theta}}[\Psi(\theta) ; A(x)]\right| \leq\left\|\mathrm{I}_{A(x)} \mid\right\|_{p} \cdot\|\Psi(\theta)\|_{q}=z^{\frac{1}{p}}(\theta ; x) \cdot\|\Psi(\theta)\|_{q},
$$

and the claim follows by setting $p=1$, by taking logarithms and by letting $x \rightarrow \infty$. Therefore $z(\theta ; x)$ and $z^{\prime}(\theta ; x)$ have similar logarithmic asymptotics, as $x \rightarrow \infty$, and so an asymptotically efficient change of measure for $z(\theta ; x)$ should be asymptotically efficient for $z^{\prime}(\theta ; x)$ as well. Thus, with $A(x)=\mathrm{I}\{S \geq x\}$ the accuracy of estimator inside the expectation in (15) can be improved by a second change of measure, which shifts the mean of the distribution of $S$ towards $x$. This shift can be obtained by an exponential tilt of $\mathrm{P}_{\theta_{0}}$, precisely by the exponentially tilted measure $\tilde{P}_{\tau}$ such that

$$
\frac{\mathrm{d} \tilde{\mathrm{P}}_{\tau}}{\mathrm{dP}_{\theta_{0}}}=\exp \left\{\tau S-K_{\theta_{0}}(\tau)\right\}
$$

The real tilting parameter $\tau$ is chosen as a saddlepoint, precisely as the solution in $v$ of (6) with $\theta=\theta_{0}$, and it is again denoted $v_{x}$. Then, we obtain the score with importance sampling Monte Carlo estimator of the sensitivity by

$$
z^{\prime}(\theta ; x)=\mathrm{E}_{\tilde{\mathrm{P}}_{v_{x}}}\left[Z(x) \Psi(\theta) \frac{\mathrm{dP}_{\theta}}{\mathrm{d}_{v_{v_{x}}}}\right],
$$

where $\theta_{0}$ has now been replaced by $\theta$, as proposed by Asmussen and Rubinstein (1999), who also showed its logarithmic efficiency ${ }^{1}$.

\subsection{Comparisons for Poisson random sums}

If $N$ is Poisson distributed with parameter $\theta \in \Theta \stackrel{\text { def }}{=} \mathbb{R}_{+}^{*}$, then we find the score

$$
\Psi(\theta)=\frac{N}{\theta}-1
$$

\footnotetext{
${ }^{1}$ Assume $z(x)=\mathrm{P}[A(x)] \rightarrow 0$, as $x \rightarrow \infty$. The unbiased estimator $Z(x)=\mathrm{I}_{A(x)}$ of $z(x)$ is logarithmic efficient if$$
\liminf _{x \rightarrow \infty} \frac{|\log \operatorname{var}(Z(x))|}{\left|\log z^{2}(x)\right|} \geq 1
$$ 
In this case, the change of integration and differentiation orders, which leads to formula (15), can be justified as follows. Let us denote $g(\theta ; x)=e^{\theta} z(\theta ; x)$. From (1) we obtain the power series representation in $\theta$

$$
g(\theta ; x)=\sum_{k=0}^{\infty} \frac{\theta^{k}}{k !} \overline{F_{X}^{* k}}(x-)
$$

where $\overline{F_{X}^{* k}}=1-F_{X}^{* k}$ and $F_{X}^{* k}$ denotes the $k$ th convolution power of $F_{X}, \forall k \in \mathbb{N}$. The convergence radius of this power series is $r=\infty$, because

$$
r=\left(\limsup _{k \rightarrow \infty}\left\{\left|\frac{\overline{F^{* k}}(x-)}{k !}\right|\right\}^{\frac{k}{2}}\right)^{-1} \geq\left(\limsup _{k \rightarrow \infty}\left\{\frac{1}{k !}\right\}^{\frac{k}{2}}\right)^{-1}=\infty
$$

Hence $g(\theta ; x)$ is differentiable w.r.t. $\theta$ over $\Theta$ and

$$
g^{\prime}(\theta ; x) \stackrel{\text { def }}{=} \frac{\partial}{\partial \theta} g(\theta ; x)=\sum_{k=1}^{\infty} \frac{\theta^{k}-1}{(k-1) !} \overline{F_{X}^{* k}}(x-), \quad \forall \theta \in \Theta
$$

Thus

$$
z^{\prime}(\theta ; x)=\left(e^{-\theta}\right)^{\prime} g(\theta ; x)+e^{-\theta} g^{\prime}(\theta ; x)=\sum_{k=0}^{\infty} \frac{\partial}{\partial \theta}\left(e^{-\theta} \frac{\theta^{k}}{k !}\right) \overline{F_{X}^{* k}}(x-)=\mathrm{E}_{\mathrm{P}_{\theta}}[Z(x) \Psi(\theta)]
$$

where the score $\Psi(\theta)$ is given by (18).

Concerning the score with importance sampling Monte Carlo estimator, given inside the expectation in (17), we note the closure of the compound Poisson distribution w.r.t. exponential tilting, as

$$
K_{\theta}(v ; \tau) \stackrel{\text { def }}{=} \log \mathrm{E}_{\tilde{\mathbf{P}}_{\tau}}\left[e^{v S}\right]=\theta_{\tau}\left[M_{X}(v ; \tau)-1\right],
$$

where

$$
\theta_{\tau} \stackrel{\text { def }}{=} \theta M_{X}(\tau) \text { and } M_{X}(v ; \tau) \stackrel{\text { def }}{=} \frac{M_{X}(\tau+v)}{M_{X}(\tau)},
$$

which compares with (8).

Example 3.1.1 Poisson random sum with Gamma summands

In this example we consider $N$ Poisson distributed with parameter $\theta>0$ and $X_{1}, X_{2}, \ldots$ Gamma distributed with density

$$
f_{X}(x)=\frac{\beta^{\alpha}}{\Gamma(\alpha)} x^{\alpha-1} e^{-\beta x}, \quad \forall x>0
$$

for some parameters $\alpha, \beta>0$. In this case, $z^{\prime}(\theta ; x)$ can be directly computed by truncating the infinite sum in $(20)$ in conjunction with

$$
\overline{F_{X}^{* k}}(x-)=\frac{\Gamma(k \alpha ; \beta x)}{\Gamma(k \alpha)}, \forall k \in \mathbb{N}^{*} \stackrel{\text { def }}{=} \mathbb{N} \backslash\{0\}, \text { and } \overline{F_{X}^{* 0}}(x-)=\mathrm{I}\{x \leq 0\} \text {, }
$$


where $\Gamma(a ; x)=\int_{x}^{\infty} e^{-t} t^{a-1} \mathrm{~d} t, \forall a, x>0$. This method of evaluating $z^{\prime}(\theta ; x)$ and the corresponding numerical results are referred here as "exact". Exact results allow for comparisons between saddlepoint and Monte Carlo approximations to sensitivities.

The m.g.f. of $X_{1}$ is given by

$$
M_{X}(v)=\left(\frac{\beta}{\beta-v}\right)^{\alpha}, \forall v<\beta,
$$

and the first three derivatives required by the saddlepoint approximation to $z^{\prime}(\theta ; x)$, see Section 2.1, are straightforward. From (9) and (25) we obtain the saddlepoint explicitly as

$$
v_{x}=\beta-\left(\frac{\theta \alpha \beta^{\alpha}}{x}\right)^{\frac{1}{1+\alpha}}
$$

The details for computing the score with importance sampling Monte Carlo estimator (17) are as follows. From (22) and (25) we see that under $\tilde{\mathrm{P}}_{v_{x}}$, the summands are again Gamma distributed with parameters $\alpha_{x} \stackrel{\text { def }}{=} \alpha$ and $\beta_{x} \stackrel{\text { def }}{=} \beta-v_{x}$, for $v_{x}$ given by (26). Also, the summation index is Poisson distributed with parameter $\theta_{x} \stackrel{\text { def }}{=} \theta\left\{\beta /\left(\beta-v_{x}\right)\right\}^{\alpha}$. With the c.g.f. (8), the m.g.f. (25) and the Radon-Nikodym derivative (16), we thus obtain the specific form of $(17)$ as

$$
z^{\prime}(\theta ; x)=\mathrm{E}_{\tilde{\mathrm{P}}_{v_{x}}}\left[\left(\frac{N}{\theta}-1\right) \exp \left\{-v_{x} S+\theta_{x}-\theta\right\} ; S \geq x\right] .
$$

Next, differentiating (3) yields the normal approximation to the sensitivity $z_{\mathrm{a}}^{\prime}(\theta ; x) \stackrel{\text { def }}{=}$ $\partial /(\partial \theta) z_{\mathrm{a}}(\theta ; x)=\left(\mu_{2} \theta\right)^{-1 / 2} \phi\left(\left\{\mu_{2} \theta\right\}^{-1 / 2}\{x-\mu \theta\}\right)(\mu+\{x-\mu \theta\} /\{2 \theta\})$, where $\mu=\alpha / \beta$ and $\mu_{2}=\alpha(\alpha+1) / \beta^{2}$.

In Figure 1 we compare the saddlepoint approximation with the score with importance sampling approximation to the sensitivity. We consider $\theta=1, \alpha=2, \beta=1$ and $x \in[0,20]$. Importance sampling is considered for $x>2$ only. For $x \leq 2$, we consider the simple score Monte Carlo estimator, which appears accurate over this region. The upper graphs show approximated and exact sensitivities, the central graphs show the errors and the lower graphs show the relative errors. We can see that for small upper tail probabilities (which are given in the upper axes), the saddlepoint and the score with importance sampling approximations are very accurate, as they have very small relative errors. For central probabilities, the saddlepoint approximation is less accurate. As mentioned at the beginning of Section 2, Lugannani and Rice's saddlepoint approximation can be misleading around the center of the distribution. However, small upper tail probabilities are the most relevant ones in practical situations and the saddlepoint approximation to these probabilities is very accurate. Figure 1 provides also evidence that the normal approximation is not accurate and thus it confirms the general explanations given just after (3).

In Figure 2 we compare again the saddlepoint and the score with importance sampling approximations and we consider $x=10.25, \alpha=2, \beta=1$ and $\theta \in(0,14]$. Importance sampling is considered for $\theta<5.125$ only. For $\theta \geq 5.125$ the simple score Monte Carlo estimator is considered. Again, we can note the very high accuracy of the saddlepoint 
approximation and of the score method with importance sampling. The upper graphs show approximated and exact sensitivities, the central graphs show the errors and the lower graphs show the relative errors.

The proposed condition for applying importance sampling is $\mathrm{E}_{\mathrm{P}_{\theta}}[S]<x$, i.e. $\alpha \theta / \beta<x$, within the ranges of $x$ and $\theta$ considered. Importance sampling does not bring improvements when $\mathrm{E}_{\mathrm{P}_{\theta}}[S] \geq x$ and in fact it even decreases the accuracy for values of $x$ close to 0 . This is due to the steepness of the saddlepoint curve in the vicinity of 0 . In all figures, the error is defined as $\tilde{z}^{\prime}-z^{\prime}$ and the relative error as $\left(\tilde{z}^{\prime}-z^{\prime}\right) / z^{\prime}$, where $\tilde{z}^{\prime}$ is any approximation to the sensitivity $z^{\prime}$. In Figures 1 and 2, the number of simulations is always $10^{5}$.

Example 3.1.2 Poisson random sum with Weibull summands

Let $Y$ be an exponential random variable with parameter equal to 1 and $\alpha>0$, then $Y^{1 / \alpha}$ has the Weibull distribution with parameter $\alpha$. In this example we assume $N$ Poisson distributed with parameter $\theta>0$ and $X_{1}, X_{2}, \ldots$ Weibull distributed with density

$$
f_{X}(x)=\alpha x^{\alpha-1} \exp \left\{-x^{\alpha}\right\}, \quad \forall x>0 .
$$

Now we are not able to compute the exact upper tail probabilities by (19) and the exact sensitivities by the penultimate formula in (20), so we consider an extended Monte Carlo evaluation of these values based on $10^{7}$ simulations as surrogate for the exact values.

We can easily compute the moments

$$
\mu_{k}=\mathrm{E}\left[X_{1}^{k}\right]=\Gamma\left(1+\frac{k}{\alpha}\right), \forall k \in \mathbb{N}^{*}
$$

The m.g.f. of the Weibull distribution $M_{X}(v)=\mathrm{E}\left[e^{v X_{1}}\right]$ exists for all $v$ over a neighborhood of zero iff $\alpha \geq 1$. With other words, $F_{X}$ is light-tailed iff $\alpha \geq 1$. Indeed by a change of variable, $M_{X}(v)=\int_{0}^{1} \exp \left\{v x^{1 / \alpha}\right\} e^{-x} \mathrm{~d} x+\int_{1}^{\infty} \exp \left\{v x^{1 / \alpha}\right\} e^{-x} \mathrm{~d} x$. For $\alpha \geq 1$, the integrand of the second integral is smaller or equal to $e^{-(1-v) x}$, so $M_{X}(v)$ exists $\forall v<1$ and therefore $\alpha \geq 1$ is sufficient for light-tailness. Therefore, the power series $\sum_{k=0}^{\infty} \mu_{k} v^{k} / k$ ! converges uniformly and it is also equal to $M_{X}(v), \forall v<1$. Moreover, $M_{X}(v)$ has derivatives of all orders $\forall v<1$ and they can be obtained by differentiating the series term by term. So we have

$$
M_{X}^{(l)}(v)=\sum_{k=0}^{\infty} \Gamma\left(1+\frac{l+k}{\alpha}\right) \frac{v^{k}}{k !}, \quad \forall v<1 \text { and } l \in \mathbb{N},
$$

with $M_{X}^{(0)}=M_{X}$. Thus the saddlepoint approximation to $z^{\prime}(\theta ; x)$ of Section 2.1 can be directly computed. The saddlepoint is obtained by solving (9) with (28). In this example we only apply the score Monte Carlo estimator to the sensitivity given by (15) with the score (18). We compare the Monte Carlo estimator based on $10^{5}$ simulation with the saddlepoint approximation.

In Figure 3 we consider $x \in[0,6], \theta=1$ and $\alpha=3$. The upper graphs show approximations to sensitivities, the central graphs show the errors and the lower graphs show the relative errors. The saddlepoint approximation is very accurate for small upper tail probabilities but it becomes misleading around the center and over the left part of the domain. 
Figure 1: Saddlepoint and score with importance sampling approximations to sensitivities of upper tail probabilities for the Poisson random sum with Gamma summands. Exact upper tail probabilities are indicated in the upper horizontal axes. $\theta=1, \alpha=2$ and $\beta=1$. Importance sampling for $x>2$ only.
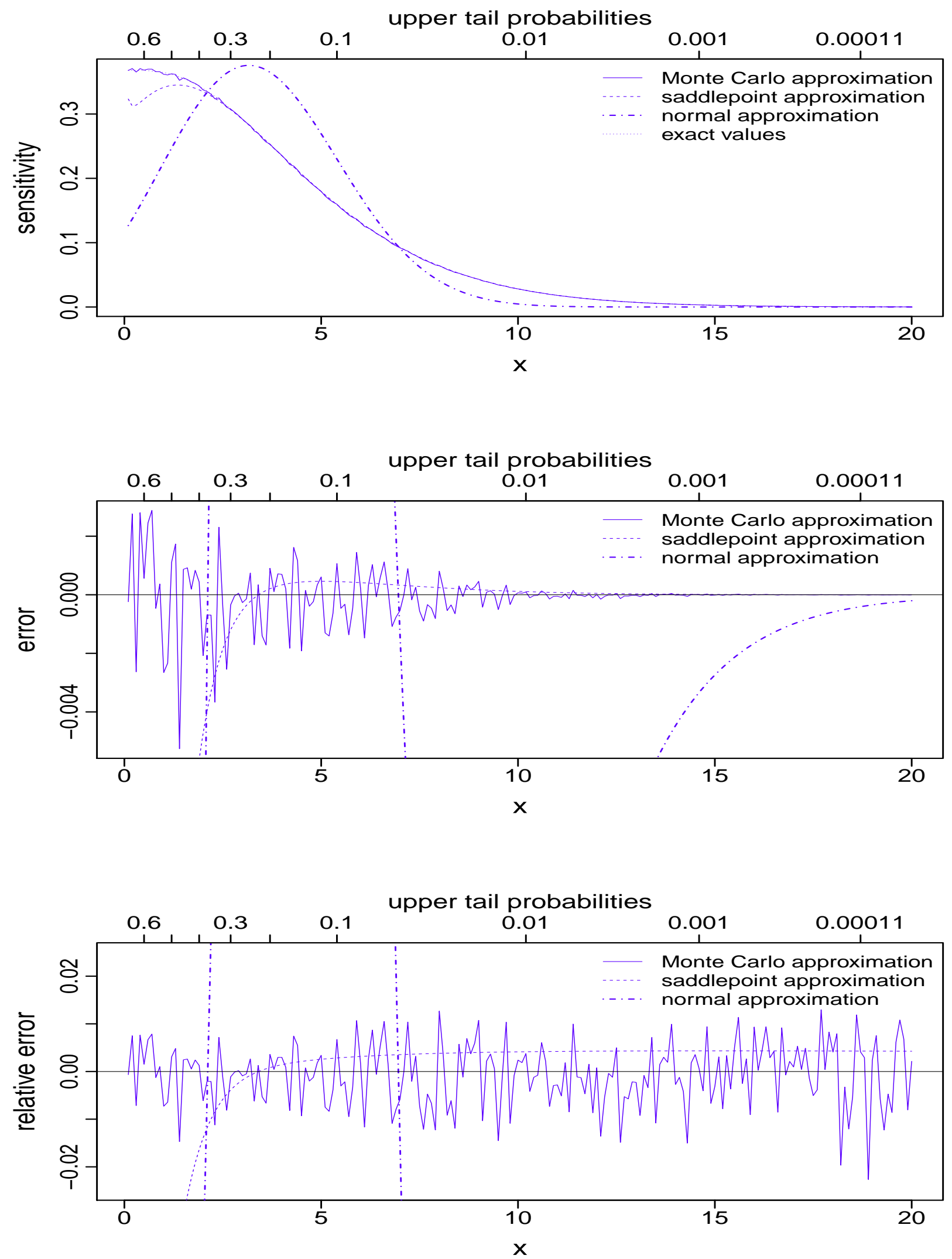
Figure 2: Saddlepoint and score with importance sampling approximations to sensitivities of upper tail probabilities for the Poisson random sum with Gamma summands. Exact upper tail probabilities are indicated in the upper horizontal axes. $x=10.25, \alpha=2$ and $\beta=1$. Importance sampling for $\theta<5.125$ only.
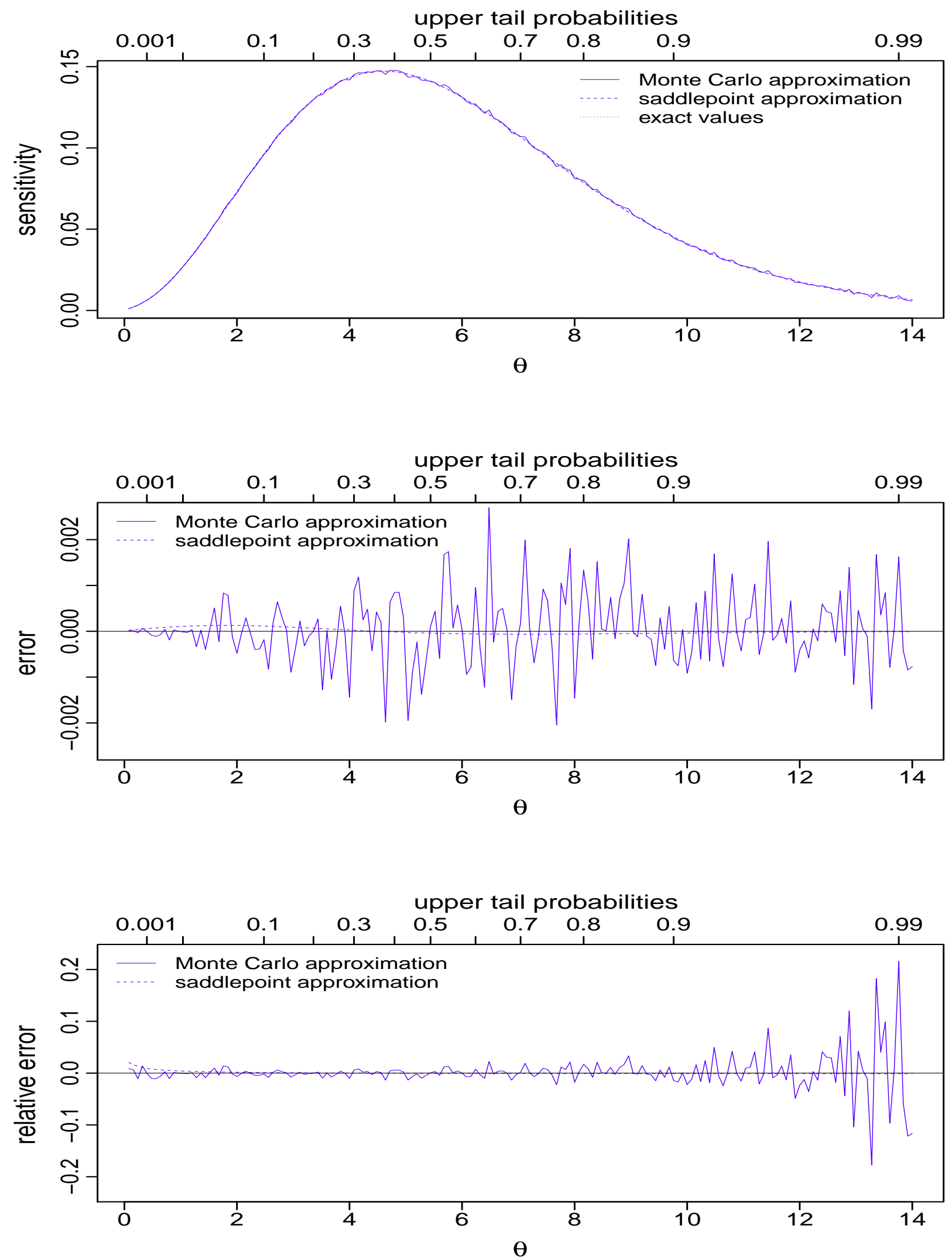
The score Monte Carlo estimator with $10^{5}$ simulations shows a substantial relative error when approximating sensitivities of very small upper tail probabilities.

In Figure 4 we consider $x=3.8, \theta \in(0,15]$ and $\alpha=3$. The upper graphs show the approximated and the exact sensitivities, the central graphs show the errors and the lower graphs show the relative errors. We can see that the saddlepoint approximation is very accurate and that the score Monte Carlo estimator based on $10^{5}$ simulations is not sufficiently accurate for approximating very small upper tail probabilities.

\subsection{Comparisons for geometric random sums}

If $N$ has the geometric distribution with parameter $\theta \in \Theta \stackrel{\text { def }}{=}(0,1)$, then we find the score

$$
\Psi(\theta)=\frac{1}{\theta}-\frac{N}{1-\theta} .
$$

We can again justify the change of integration and differentiation orders, which leads to the score Monte Carlo estimator given inside the expectation of (15), as follows. Let us denote $g(\theta ; x)=z(\theta ; x) / \theta$. From (1) we obtain the power series in $\theta$

$$
g(\theta ; x)=\sum_{k=0}^{\infty}(-1)^{k}(\theta-1)^{k} \overline{F_{X}^{* k}}(x-)
$$

The convergence radius of this power series is $r=1$, because

$$
r=\left(\limsup _{k \rightarrow \infty}\left\{\left|(-1)^{k} \overline{F_{X}^{* k}}(x-)\right|\right\}^{\frac{k}{2}}\right)^{-1} \geq\left(\limsup _{k \rightarrow \infty}\{1\}^{\frac{k}{2}}\right)^{-1}=1 .
$$

Hence $g(\theta ; x)$ is differentiable w.r.t. $\theta$ over $\Theta$ and

$$
g^{\prime}(\theta ; x) \stackrel{\text { def }}{=} \frac{\partial}{\partial \theta} g(\theta ; x)=\sum_{k=1}^{\infty}(-1)^{k} k(\theta-1)^{k-1} \overline{F_{X}^{* k}}(x-), \quad \forall \theta \in \Theta .
$$

Thus from previous developments,

$$
z^{\prime}(\theta ; x)=\theta^{\prime} g(\theta ; x)+\theta g^{\prime}(\theta ; x)=\sum_{k=0}^{\infty} \frac{\partial}{\partial \theta} \theta(1-\theta)^{k} \overline{F_{X}^{* k}}(x-)=\mathrm{E}_{\mathrm{P}_{\theta}}[Z(x) \Psi(\theta)],
$$

where the score $\Psi(\theta)$ is given by (29).

Regarding the score with importance sampling estimator (17), we note the closure of the compound Geometric distribution w.r.t. exponential tilting, as

$$
K_{\theta}(v ; \tau) \stackrel{\text { def }}{=} \log \mathrm{E}_{\tilde{\mathrm{P}}_{\tau}}\left[e^{v S}\right]=\log \frac{\theta_{\tau}}{1-\left(1-\theta_{\tau}\right) M_{X}(v ; \tau)}
$$

where

$$
\theta_{\tau} \stackrel{\text { def }}{=} 1-(1-\theta) M_{X}(\tau) \text { and } M_{X}(v ; \tau) \stackrel{\text { def }}{=} \frac{M_{X}(\tau+v)}{M_{X}(\tau)},
$$

which compares with (11). 
Figure 3: Saddlepoint and score Monte Carlo approximations to sensitivities of upper tail probabilities for the Poisson random sum with Weibull summands. Exact upper tail probabilities are indicated in the upper horizontal axes. $\theta=1$ and $\alpha=3$.
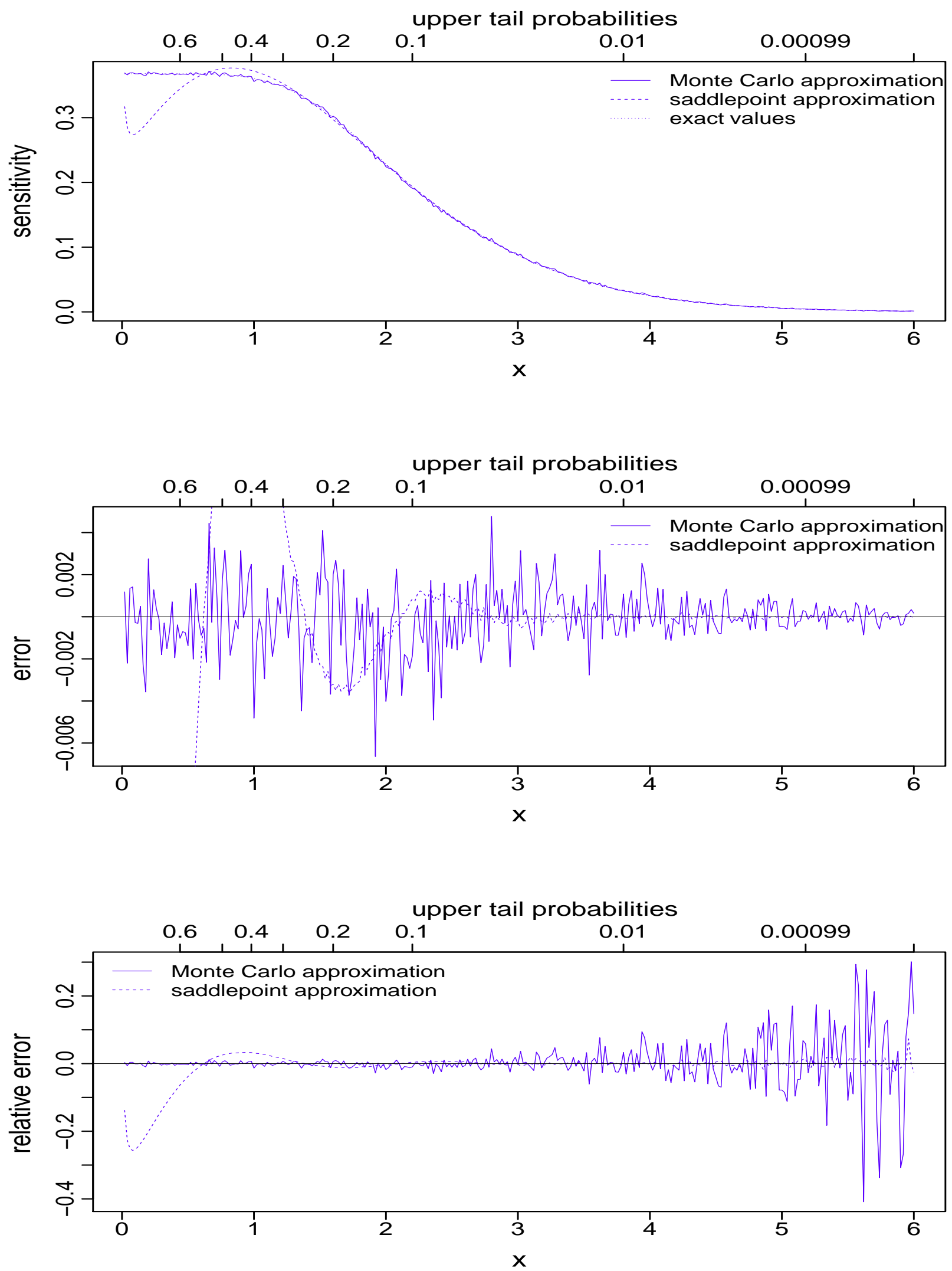
Figure 4: Saddlepoint and score Monte Carlo approximations to sensitivities of upper tail probabilities for the Poisson random sum with Weibull summands. Exact upper tail probabilities are indicated in the upper horizontal axes. $x=3.8$ and $\alpha=3$.
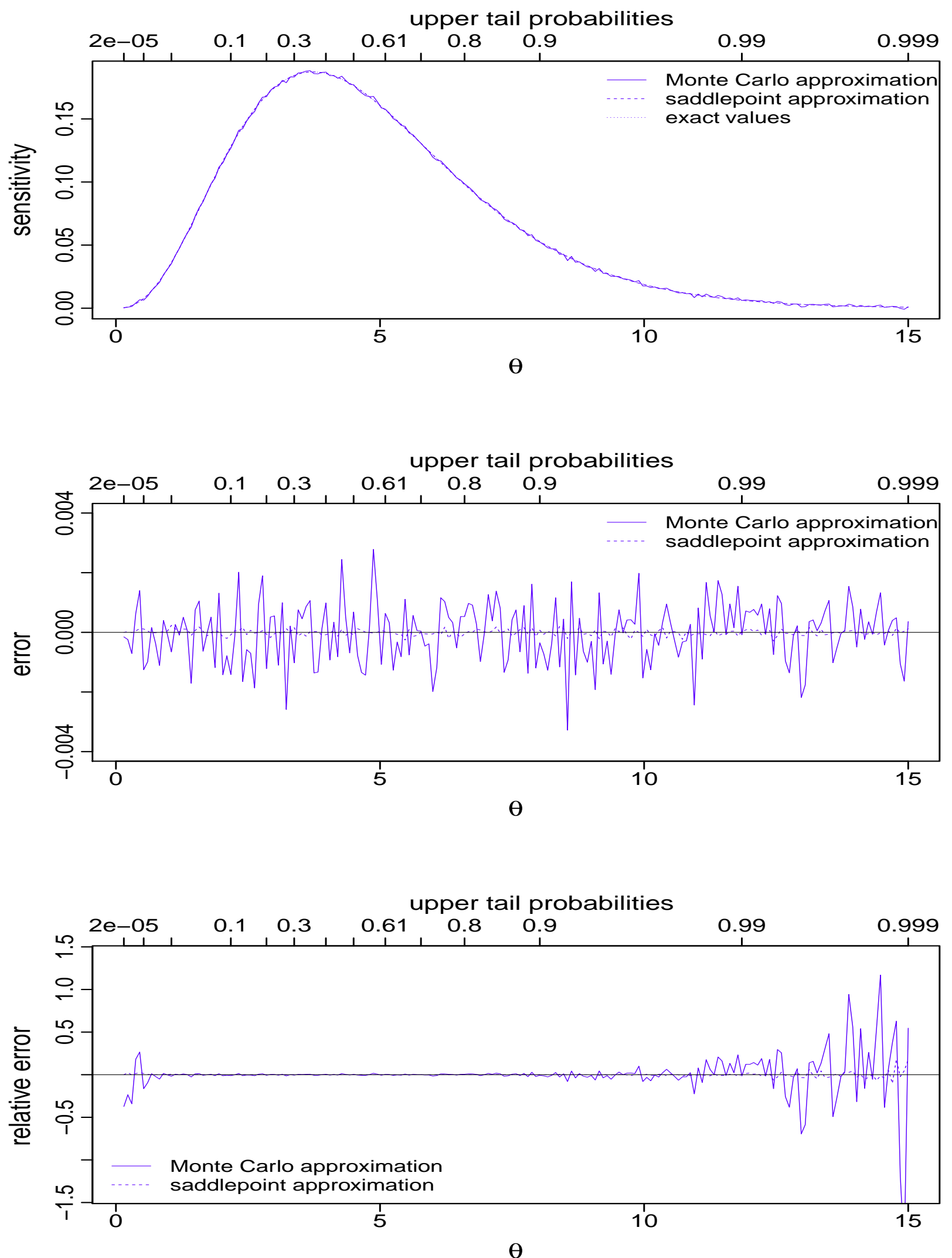
Example 3.2.1 Geometric random sum with Gamma summands

In this example $N$ has the Geometric distribution with parameter $\theta \in(0,1)$ and $X_{1}, X_{2}, \ldots$ are Gamma distributed with density (23) and m.g.f. (25). In this case, $z^{\prime}(\theta ; x)$ can be directly computed by truncating the infinite sum in (30) in conjunction with the convolution formula (24) of the Gamma distribution. In this way we obtain exact values which allow for comparisons.

The m.g.f. of $X_{1}$ is given by (25) and the saddlepoint approximation to $z^{\prime}(\theta ; x)$ can be directly obtained with the results of Section 2.2. In particular, we obtain from (14) and (25) that, $\forall x>0$, the saddlepoint $v_{x}$ is the solution in $v$ of

$$
-x(\beta-v)^{1+\alpha}+x \beta^{\alpha}(1-\theta)(\beta-v)+\alpha \beta^{\alpha}(1-\theta)=0
$$

which satisfies $M_{X}(v)<1 /(1-\theta)$, i.e. the solution in $v$ such that $v<\beta\left\{1-(1-\theta)^{1 / \alpha}\right\}$. When $\alpha$ is an integer, (33) is a polynomial of degree $1+\alpha$ in the variable $w=\beta-v$. For $\alpha=2$ or 3 , (33) is a cubic or a quartic and so its roots can be found algebraically. However no general formulae for the roots are available for degrees 5 or higher. Generally, one could apply the method of Weierstrass, also called method of Durand-Kerner, which finds iteratively the roots of polynomials of any degree; see e.g. Dahlquist and Björck (2008), Section 6.5.4. Alternatively, one can apply general root finder algorithms. The score with importance sampling estimator (17) follows from the following computations. Under $\tilde{\mathrm{P}}_{v_{x}}$, the summands are Gamma distributed with parameters $\alpha_{x}=\alpha$ and $\beta_{x}=\beta-v_{x}$. The summation index has the Geometric distribution with parameter $\theta_{x}=1-(1-\theta)\{\beta /(\beta-$ $\left.\left.v_{x}\right)\right\}^{\alpha}$. With the c.g.f. (11), the m.g.f. (25) and the Radon-Nikodym derivative (16), we obtain the specific form of (17) as

$$
z^{\prime}(\theta ; x)=\mathrm{E}_{\tilde{\mathrm{P}}_{v_{x}}}\left[\left(\frac{1}{\theta}-\frac{N}{1-\theta}\right) \exp \left\{-v_{x} S\right\} \frac{\theta}{\theta_{x}} ; S \geq x\right] .
$$

For $\theta=0.5, \alpha=2$ and $\beta=1$, the domain of existence of the saddlepoint, defined by $(33)$, is $\left(-\infty, \beta\left\{1-(1-\theta)^{1 / \alpha}\right\}\right)=(-\infty, 1-1 / \sqrt{2}) \simeq(-\infty, 0.2929)$. Thus (33) is the cubic $-x w^{3}+(x / 2) w+1=0$, where $w=1-v$. Every cubic has at least one real solution and the following cases can be distinguished. Let $a w^{3}+b w^{2}+c w+d=0$, where $a, b, c, d \in \mathbb{R}$, and $\Delta=18 a b c d-4 b^{3} d+b^{2} c^{2}-4 a c^{3}-27 a^{2} d^{2}$. If $\Delta>0$, then the cubic has three distinct real roots. If $\Delta=0$, then the cubic has a multiple root and all its roots are real. If $\Delta<0$, then the cubic has one real root and two nonreal complex conjugate roots. In our case $\Delta=x^{2}\left(x^{2} / 2-27\right)$, which has the double root $x=0$ and two simple roots $x= \pm \sqrt{54} \simeq \pm 7.3485$. Thus the cubic has one real root and two nonreal complex conjugate roots $\forall x \in(0, \sqrt{54})$ and three distinct real roots $\forall x \in(\sqrt{54}, \infty)$. Over these two last intervals, $v_{x}$ is the unique real root smaller than $1-1 / \sqrt{2} \simeq 0.2929$.

In Figure 5 we compare the saddlepoint approximation with the score with importance sampling Monte Carlo approximation to the sensitivity. We consider $\theta=0.5, \alpha=2, \beta=1$ and $x \in[0,40]$. Importance sampling is considered for $x>2$ only. The upper graphs show the approximated and the exact sensitivities, the central graphs show the errors and the relative errors are shown by the lower graphs. The saddlepoint approximation is very accurate for small upper tail probabilities, although the score with importance sampling 
Monte Carlo method seems more accurate. Here also, the accuracy of the saddlepoint approximation deteriorates around the center of the distribution.

In Figure 6 we compare again the saddlepoint approximation and the score with importance sampling estimator and we now consider $x=14, \alpha=2, \beta=1$ and $\theta \in(0,1)$. Importance sampling is considered for $\theta>0.125$ only. The upper graphs show approximated and exact sensitivities, the central graphs show errors and relative errors are shown in the lower graphs. The saddlepoint and the score with importance sampling approximations appear very accurate. However, the lower graphs of Figure 8 indicate that the relative error of the saddlepoint approximation increases as $\theta$ approches 1 , where the corresponding upper tail probabilities become extremely small. This can be explained as follows. From $\mathrm{E}_{\mathrm{P}_{\theta}}[N]=(1-\theta) / \theta \rightarrow 0$, as $\theta \rightarrow 1$, a vanishing number of summands is expected in the random sum $S$ and this fact affects the accuracy of the saddlepoint approximation, which is asymptotic in the sense that the relative error tends to zero as the expected number of summands tends to infinity.

The restrictions on the regions of importance sampling result from $\mathrm{E}_{\mathrm{P}_{\theta}}[S]<x$ i.e. $(1-\theta) / \theta \cdot \alpha / \beta<x$. The number of simulations in Figures 5 and 6 is $10^{5}$.

\section{Conclusion}

In the previous sections we showed how sensitivities of upper tail probabilities of random sums with Poisson or Geometric distributed summation indices and Gamma or Weibull distributed summands can be obtained by saddlepoint approximations. The relative errors entailed the saddlepoint approximations are very small and similar in magnitude to the ones entailed by the score with importance sampling Monte Carlo method.

Measurements of computing times of selected sensitivities in the settings of Examples 3.1.1 and 3.2.1 are given in Table 1. All computations in this article were done with $\mathrm{R}$ ( $\mathrm{R}$ Development Core Team, 2012) and the times displayed in Table 1 are averages of 10 measurements obtained by the third output of the $\mathrm{R}$ function system.time, which measures the real elapsed time from the beginning until the end of a process. In the case of the Poisson sum with Gamma summands of Example 3.1.1, the computation time of the saddlepoint approximation is almost null, because the saddlepoint is defined explicitly by (26). In the case of the Geometric sum with Gamma summands of Example 3.2.1, the computation time of the saddlepoint approximation is larger, because the saddlepoint is computed iteratively by solving (33). But this time is still 10 times smaller than by score with importance sampling. Another practical advantage of the saddlepoint approximation is that it yields sensitivity curves which are continuous w.r.t. the parameter values or the abscissa values of the d.f.

The following recommendations can be drawn from this numerical study.

- Sensitivities of small or very small upper tail probabilities can be efficiently computed by the saddlepoint approximation. Score with importance sampling is an alternative accurate method for these situations, but its computing time is substantially higher.

- For sensitivities of central probabilities, both the proposed saddlepoint approximation 
Figure 5: Saddlepoint and score with importance sampling approximations to sensitivities of upper tail probabilities for the Geometric random sum with Gamma summands. Exact upper tail probabilities are indicated in the upper horizontal axes. $\theta=0.5, \alpha=2$ and $\beta=1$. Importance sampling for $x>2$ only.
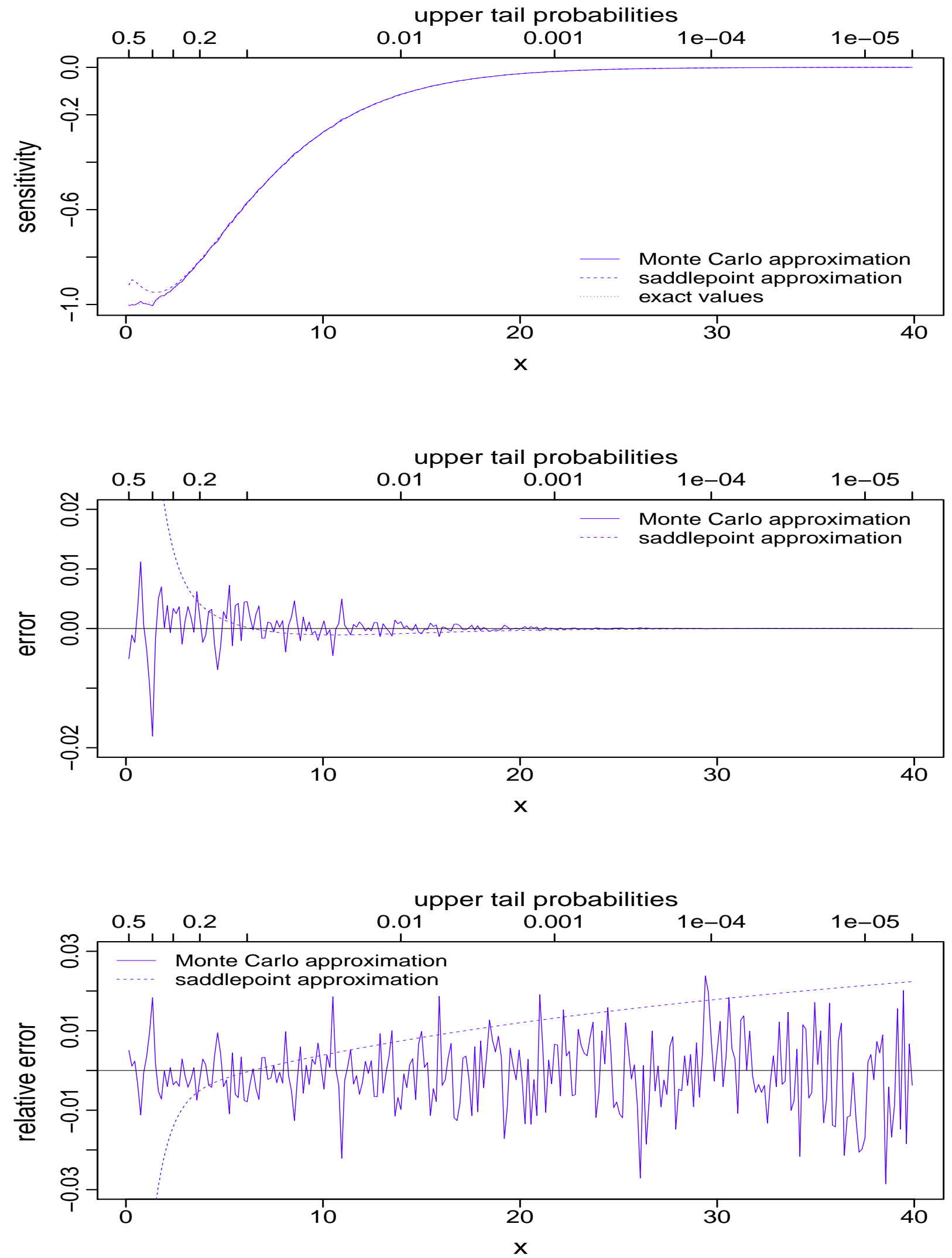
Figure 6: Saddlepoint and score with importance sampling approximations to sensitivities of upper tail probabilities for the Geometric random sum with Gamma summands. Exact upper tail probabilities are indicated in the upper horizontal axes. $x=14, \alpha=2$ and $\beta=1$. Importance sampling for $\theta>0.125$ only.
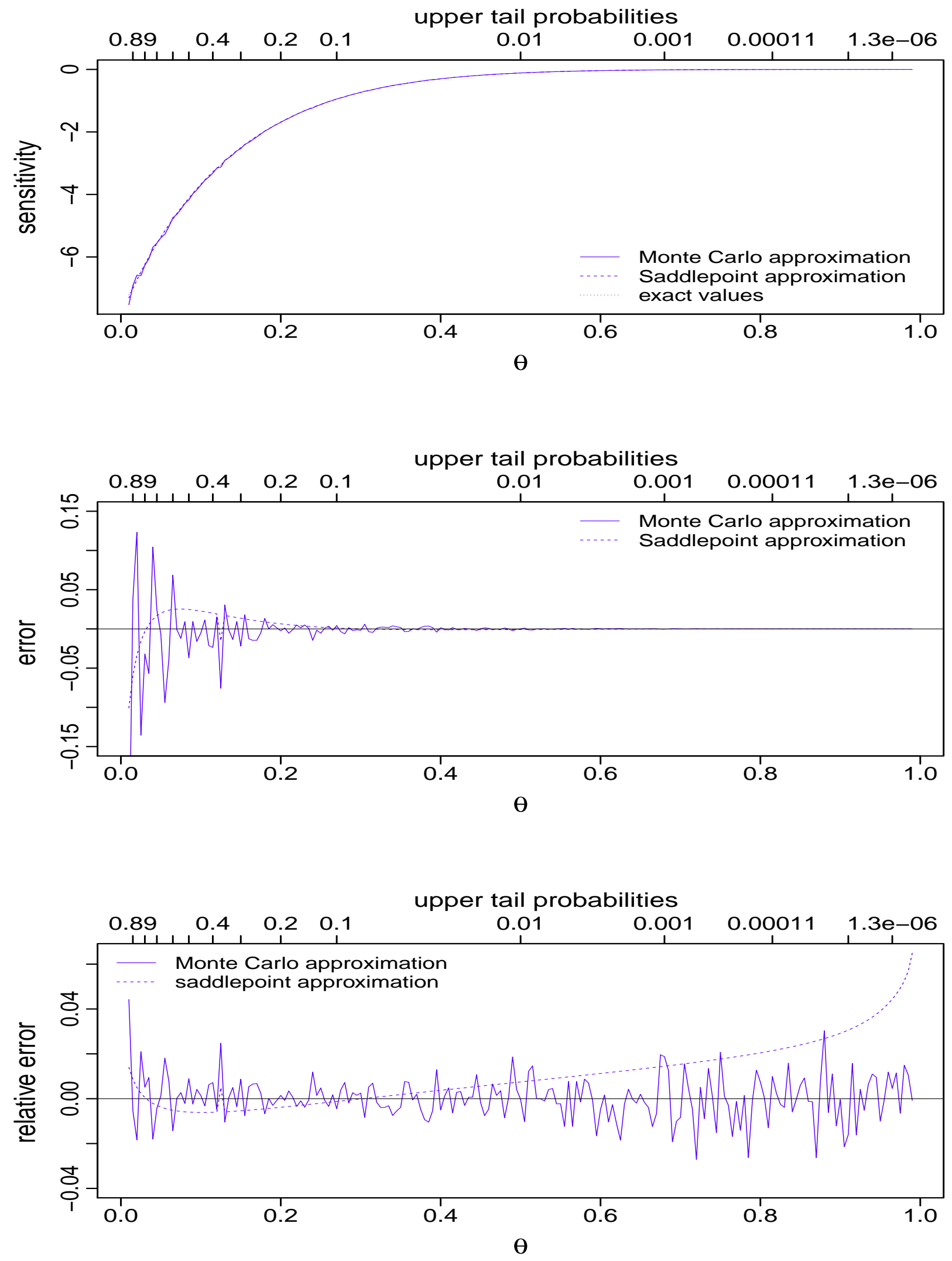
Table 1: Measurement A: Example 3.1.1, Poisson $(\theta=1)$, Gamma $(\alpha=2, \beta=1)$. Measurement B: Example 3.2.1, Geometric $(\theta=1 / 2)$, Gamma $(\alpha=2, \beta=1)$. $t_{\text {is }}$ : computung time in seconds for score with importance sampling. $t_{\mathrm{s}}$ : computing time in seconds for saddlepoint approximation.

\begin{tabular}{|c|r|r|r|r|}
\hline Measurement & $x$ & $z(\theta ; x)$ & $t_{\text {is }}$ & $t_{\mathrm{s}}$ \\
\hline \hline $\mathrm{A}$ & 8.80 & 0.02062 & 1.771 & 0.000 \\
& 11.60 & 0.00505 & 1.754 & 0.000 \\
\hline $\mathrm{B}$ & 11.55 & 0.02049 & 1.771 & 0.176 \\
& 16.35 & 0.00502 & 1.805 & 0.183 \\
\hline
\end{tabular}

and the score method with importance sampling should be avoided. The simple Monte Carlo score method should be preferred in these situations.

The kind of accuracy of the saddlepoint approximation observed in Section 3 is expected with other light-tailed summand or summation index distributions. Neither the saddlepoint approximation nor the score with importance sampling method can be applied with heavytailed distributions. In this case, either the simple score Monte Carlo method or alternative Monte Carlo methods presented in Chapter VII of Asmussen and Glynn (2007) could be applied, although they may not lead to small relative errors or to high computational speed, as with the saddlepoint approximation or with the score with importance sampling method.

One could also approximate sensitivities by the Fast Fourier transform (FFT). Gatto and Mosimann (2012) present numerical comparisons of the saddlepoint approximation with various numerical methods, including importance sampling and FFT, for approximating the probability of ruin of the risk process (which is thus the upper tail probability of a geometric sum, see Section 1). In their study, the saddlepoint approximation and importance sampling are the most accurate for approximating very small probabilities of ruin.

The $\mathrm{R}$ programs of the approximations presented is available at http://www.stat. unibe.ch.

\section{References}

Asmussen S. (2000), Ruin Probabilities, World Scientific.

Asmussen S., Glynn P. W. (2007), Stochastic Simulation. Algorithms and Analysis, Springer.

Asmussen, S., Rubinstein, R. Y. (1999), "Sensitivity analysis of insurance risk models via simulation", Management Science, 45, 1125-1141.

Dahlquist, G., Björk, A. (2008), Numerical methods in scientific computing, Volume I, SIAM, Philadelphia.

Daniels, H. E. (1954), "Saddlepoint approximations in statistics", Annals of Mathematical Statistics, 25, 631-650.

Daniels, H. E. (1987), "Tail probability approximations", International Statistical Review, $55,37-48$. 
De Bruijn, N. G. (1981), Asymptotic methods in analysis, Dover.

Embrechts, P., Jensen, J. L., Maejima, M., Teugels, J. L. (1985), "Approximations for compound Poisson and Pólya processes", Advances in Applied Probability, 17, 623-637.

Esscher, F. (1932), "On the probability function in the collective theory of risk", Skandinavisk Aktuarietidskrift, 175-195.

Esscher, F. (1963), "On approximate computations when the corresponding characteristic functions are known", Skandinavisk Aktuarietidskrift, 78-86.

Gatto, R. (2011), "Values and tail values at risk of doubly compound inhomogeneous and contagious aggregate loss processes", Mathematical and Computer Modelling, 54, 15231535 .

Gatto, R. (2012), "Saddlepoint approximations to tail probabilities and quantiles of inhomogeneous discounted compound Poisson processes with periodic intensity functions", Methodology and Computing in Applied Probability, 14, 1053-1074.

Gatto, R., Mosimann, M. (2012), "Four approaches to compute the probability of ruin in the compound Poisson risk process with diffusion", Mathematical and Computer Modelling, 55, 1169-1185.

Jensen, J. L. (1991), "Saddlepoint approximations to the distribution of the total claim amount in some recent risk models", Scandinavian Actuarial Journal, 2, 154-168.

Lugannani, R., Rice, S. (1980), "Saddle point approximation for the distribution of the sum of independent random variables", Advances in Applied Probability, 12, 475-490.

R Development Core Team (2012), R: A Language and Environment for Statistical Computing, R Foundation for Statistical Computing, Vienna (Austria).

Rubinstein, R. Y. (1986), "The score function approach for sensitivity analysis of computer simulation models", Mathematics and Computers in Simulation, 28, 351-379.

Willmot, G. E. (1989), "The total claims distribution under inflationary conditions", Scandinavian Actuarial Journal, 1, 1-12. 